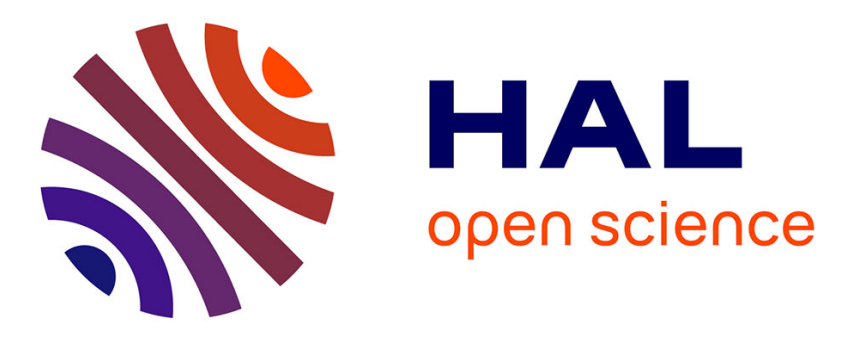

\title{
Structure-directing role of immobilized polyoxometalates in the synthesis of porphyrinic Zr-based metal-organic frameworks
}

Mathis Duguet, Alex Lemarchand, Youven Benseghir, Pierre Mialane, Maria Gomez-Mingot, Catherine Marchal-Roch, Mohamed Haouas, Marc Fontecave, Caroline Mellot-Draznieks, Capucine Sassoye, et al.

\section{To cite this version:}

Mathis Duguet, Alex Lemarchand, Youven Benseghir, Pierre Mialane, Maria Gomez-Mingot, et al.. Structure-directing role of immobilized polyoxometalates in the synthesis of porphyrinic Zr-based metal-organic frameworks. Chemical Communications, 2020, 56 (70), pp.10143-10146. 10.1039/d0cc04283h . hal-02945355

\section{HAL Id: hal-02945355 https://hal.science/hal-02945355}

Submitted on 28 Sep 2020

HAL is a multi-disciplinary open access archive for the deposit and dissemination of scientific research documents, whether they are published or not. The documents may come from teaching and research institutions in France or abroad, or from public or private research centers.
L'archive ouverte pluridisciplinaire HAL, est destinée au dépôt et à la diffusion de documents scientifiques de niveau recherche, publiés ou non, émanant des établissements d'enseignement et de recherche français ou étrangers, des laboratoires publics ou privés. 


\title{
Structure-directing role of immobilized polyoxometalates in porphyrinic Zr-based Metal-Organic Frameworks synthesis
}

\author{
Mathis Duguet, ${ }^{\mathrm{a}, \mathrm{b}}$ Alex Lemarchand, ${ }^{\mathrm{b}}$ Youven Benseghir, ${ }^{\mathrm{a}, \mathrm{b}}$ Pierre Mialane, ${ }^{\mathrm{a}}$ Maria Gomez-Mingot, \\ Catherine Roch-Marchal, ${ }^{a}$ Mohamed Haouas, ${ }^{a}$ Marc Fontecave, ${ }^{\text {b }}$ Caroline Mellot-Draznieks, ${ }^{* b}$ \\ Capucine Sassoye*c and Anne Dolbecq*a
}

\begin{abstract}
We evidence the structure-directing role of the $\mathrm{PW}_{12} \mathrm{O}_{40}{ }^{3-}$ polyoxometalate in the synthesis of porphyrinic MOFs whereby it promotes the formation of the kinetic topology. Its immobilization into the MOF is successfully achieved at high temperature yielding the kinetic MOF-525/PCN-224 phases, while prohibiting the formation of the thermodynamic MOF-545 product. A combined experimental and theoretical approach uses differential PDF and DFT calculations along with solid-state NMR to show the structural integrity of the hosted POM and its location in the vicinity of the $\mathrm{Zr}$ based nodes.
\end{abstract}

Over the years, Metal-Organic Frameworks (MOFs) have been extensively studied for a variety of applications such as gas storage, drug delivery or catalysis. ${ }^{1-3}$ In the strategic field of solar energy conversion, the use of porphyrinic $\mathrm{Zr}_{6}$-TCCP-based MOFs constructed from $\mathrm{Zr}_{6} \mathrm{O}_{4}(\mathrm{OH})_{4}$ inorganic nodes and tetrakis(4-carboxyphenyl)porphyrin (TCPP) linkers for photo- or electrocatalysis purposes has attracted an ever-growing interest, knowing the porphyrin can act both as a photosensitizer and/or catalyst when metallated. 4,5 Amongst this subfamily, three topologies have attracted particular attention, namely MOF-525, ${ }^{6}$ PCN-2247 and MOF-545 ${ }^{6}$ (also

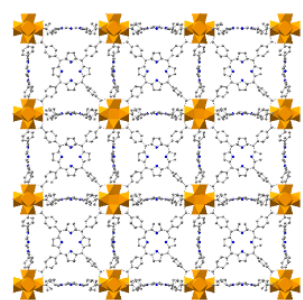

MOF-525

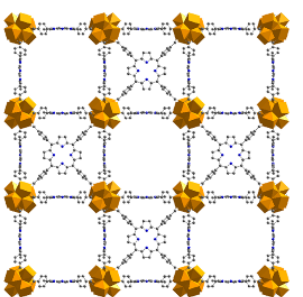

$\mathrm{PCN}-224$

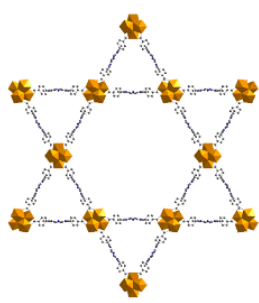

MOF-545
Fig. 1 The three porphyrinic $\mathrm{Zr}_{6}$-TCCP-based MOFs encountered in this study. MOF-525 and PCN-224 exhibit cubic cavities, PCN-224 being described as a MOF-525 with missing linkers. MOF-545 possesses hexagonal channels.

a. Université Paris-Saclay, UVSQ, CNRS, UMR 8180, Institut Lavoisier de Versailles, 78000 Versailles, France.E-mail : anne.dolbeca@uvsq.fr

b. Laboratoire de Chimie des Processus Biologiques, UMR CNRS 8229, Collège de France, Sorbonne Université, PSL Research University, 11 Place Marcelin Berthelot, 75231 Paris Cedex 05, France. E-mail: caroline.mellot-

draznieks@college-de-france.fr

Sorbonne Université, UMR 7574, Collège de France, Laboratoire de Chimie de la Matière Condensée de Paris, 4 Place Jussieu, 75252 Paris cedex 05, France. Email: capucine.sassoye@upmc.fr

tElectronic Supplementary Information (ESI) available: Detailed synthesis and characterizations. See DOI: 10.1039/x0xx00000x known as PCN-222) (Fig. 1). Due to the large size of the TCPP linker, these three $\mathrm{Zr}_{6}$-TCCP-based MOFs exhibit sufficiently wide pores and high surface areas to allow the heterogenization of molecular catalysts to host energy conversion-related reactions, an emerging sub-field. ${ }^{8}$ We thus recently showed that the immobilization of a cobalt sandwich-type polyoxometalate (POM) in MOF-545 leads to a noble metal-free heterogeneous photosystem with a high photocatalytic activity for water oxidation reaction, whereby MOF-545 acts as both a photosensitizer and an heterogenization matrix. ${ }^{9}$ POMs can also be co-immobilized with complexes in MOFs' pores in order to boost their photocatalytic activity. Recently, we evidenced that the incorporation of the $\mathrm{PW}_{12} \mathrm{O}_{40}{ }^{3-}\left(\mathrm{PW}_{12}\right)$ Keggin-type POM inside the Cp*Rh@UiO-67 MOF increased its photocatalytic activity for $\mathrm{CO}_{2}$ reduction reaction $\left(\mathrm{CO}_{2} \mathrm{RR}\right)$ when compared to that of the POM-free catalyst. ${ }^{10}$ Amongst other MOFs, ${ }^{11}$ MOF525 was shown to display significant catalytic activity for $\mathrm{CO}_{2} \mathrm{RR}$. While Hod et al. first showed the electrocatalytic properties of MOF-525(Fe), ${ }^{12}$ Zhang et al. demonstrated a year later the photocatalytic activity for $\mathrm{CO}_{2} \mathrm{RR}$ of its free-base, Co- and $\mathrm{Zn}$ metallated forms under visible light. ${ }^{13}$ Based on these studies, we decided to attempt the immobilization of $\mathrm{PW}_{12}$ inside Femetallated $\mathrm{Zr}_{6}$-TCPP-based MOFs in view of modifying their catalytic properties at a later stage. It must be recalled that the targeted synthesis of these phases is still the subject of intense research. ${ }^{12-18}$ For example, the influence of the synthetic temperature $^{14}$ or that of the modulator ${ }^{16}$ was explored, whereby MOF-525 and PCN-224 synthesized at room temperature (RT) were shown to be kinetic outcomes while MOF-545 was identified as the thermodynamic phase obtained at higher temperature. A consequence of the kinetic nature of MOF-525 and PCN-224 and of their structural similarity is that they are often obtained as a mixture, yielding very similar X-ray diffraction patterns. This led us to investigate the synthetic parameters which allow to get the desired kinetic Fe-metallated MOF-525/PCN-224 phases amongst the three possible $\mathrm{Zr}_{6}{ }^{-}$ TCCP-based MOFs products (Fig. 1), the non-metallated products being also synthesized for reference purposes.

In the present work, we thus investigate the immobilization of the $\mathrm{PW}_{12}$ POM into $\mathrm{Zr}_{6}$-TCCP-based MOFs and its impact on the obtained mixture of phases. A structuring effect of the POM is reported towards the targeted kinetic MOF-525/PCN-224 phases. A complete structural characterization of the product is 
provided by means of Pair Distribution Function (PDF) analysis along with DFT calculations.

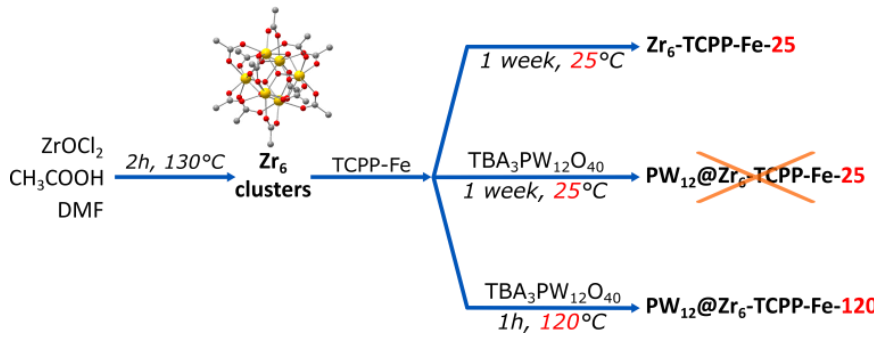

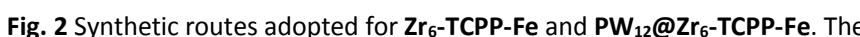
non-metallated compounds are isolated with similar procedures (see ESI+).

The synthesis of the mixed phases MOF-525/PCN-224, adapted from reported procedures, ${ }^{14,19-21}$ consists in the preparation of $\mathrm{Zr}_{6}$ clusters in solution at high temperature followed by the addition of porphyrin and stirring at RT for a week (Fig. 2). We adopted the notation suggested by Gong et al. ${ }^{14} \mathbf{Z r}_{6}-\mathrm{TCPP}-\mathrm{T}$ and $\mathbf{Z r}_{6}$-TCPP-Fe-T, $\mathbf{T}$ referring to the synthesis temperature. The powder X-ray diffraction (PXRD) diagrams of the $\mathbf{Z r}_{6}$-TCPP-25 and $\mathbf{Z r}_{6}$-TCPP-Fe-25 correspond expectedly to the simulated diagrams of the kinetic phases, MOF-525 and/or PCN-224 (Fig. $\mathrm{S} 1 \mathrm{a}, \mathrm{ESI}+)$. Regarding the immobilization of $\mathrm{PW}_{12}$ into the MOFs, a post-synthetic impregnation strategy was discarded, knowing that both PCN-224 and MOF-525 exhibit small windows which preclude the diffusion of the POM into the MOF. We thus rather adopted an in situ strategy by adding (TBA) ${ }_{3} \mathrm{PW}_{12} \mathrm{O}_{40}$ in the synthetic medium. However, RT synthesis failed at incorporating $\mathrm{PW}_{12}$ into the MOF. Increasing the synthesis temperature was found essential to allow its successful incorporation and get the targeted $\mathbf{P W}_{\mathbf{1 2}} @ \mathbf{Z r}_{6}-\mathbf{T C P P}-\mathbf{1 2 0}$ and PW $12 @ Z_{6}$-TCPP-Fe-120 obtained after $1 \mathrm{~h}$ at $120^{\circ} \mathrm{C}$. This suggests that at $120^{\circ} \mathrm{C}$ we have a fast growth of MOF-525 due to some effect of the POM at short time reaction, as detailed below. Performing the same experiment at $100^{\circ} \mathrm{C}$ allows the formation of the material but with a lower yield, while at $65^{\circ} \mathrm{C}$ an amorphous phase is obtained (Fig. S2). We focused on the impact of a stepwise increase of the amount of $\mathrm{PW}_{12}$ introduced in the reaction medium of $\mathbf{P W}_{\mathbf{1 2}} @ \mathbf{Z r}_{\mathbf{6}}$-TCPP-Fe-120 on the resulting MOFs' topologies while keeping all the other synthetic parameters constant (Fig. 3). Notably, in the absence of $\mathrm{PW}_{12}$ in the reaction medium, the high temperature synthesis of $\mathbf{Z r}_{6^{-}}$

a)

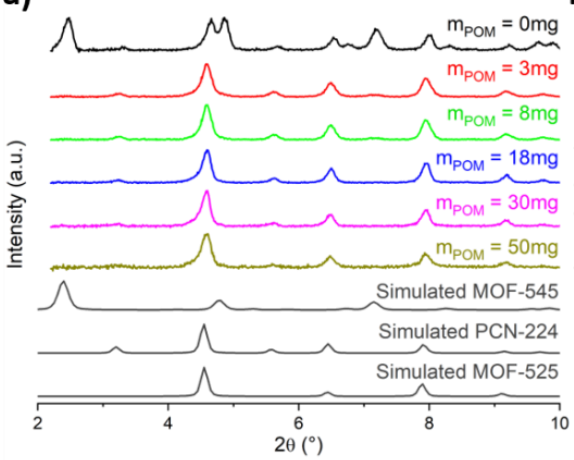

b)

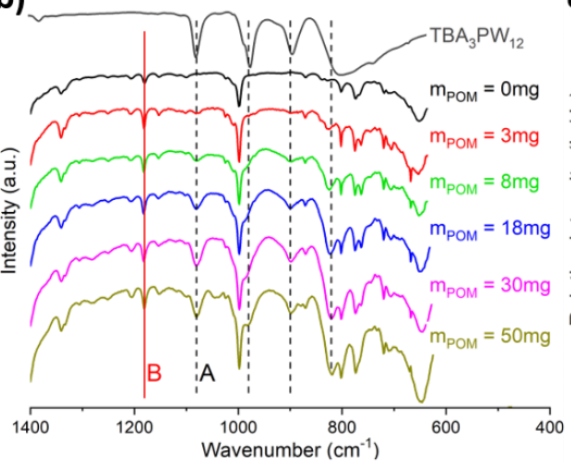

TCPP-Fe-120 leads to a mixture of the thermodynamic phase, MOF-545(Fe), and of the kinetic ones, MOF-525(Fe)/PCN224(Fe). This latter result falls in line with the temperaturetopology relationship recently reported. ${ }^{14}$ Strikingly, the formation of the thermodynamic phase MOF-545(Fe) is, however, suppressed upon the addition of a very low amount of $\mathrm{PW}_{12}$ (Fig. 3a, red), pointing towards a remarkable structuredirecting effect of $\mathrm{PW}_{12}$ in the high temperature synthesis. All the PXRD diagrams of the $\mathbf{P W}_{\mathbf{1 2}} @ \mathbf{Z r}_{\mathbf{6}}$-TCPP-Fe-120 composites obtained for higher $\mathrm{PW}_{12}$ amounts are similar, corresponding to PCN-224(Fe) or to a MOF-525(Fe)/PCN-224(Fe) mixture.

The IR spectra of this series show the unambiguous presence of the POM within all PW $12 @ Z_{\mathbf{1 2}}-\mathrm{TCPP}-\mathrm{Fe}-120$ materials, exhibiting the characteristic $\mathrm{P}-\mathrm{O}\left(1077 \mathrm{~cm}^{-1}\right), \mathrm{W}=\mathrm{O}$ $\left(972 \mathrm{~cm}^{-1}\right)$ and $\mathrm{W}-\mathrm{O}-\mathrm{W}\left(892\right.$ and $\left.797 \mathrm{~cm}^{-1}\right)$ vibration bands of $\mathrm{PW}_{12}$ (Fig. $3 \mathrm{~b}$ and Fig. S1b). In contrast we can notice the absence of aliphatic C-H vibrations in the IR spectra of the composites which indicates that no TBA counter-cations have been inserted in the matrix during the synthetic process. A semi-quantitative analysis of the IR spectra was undertaken by following the relative intensities of the bands at $1077 \mathrm{~cm}^{-1}$ attributed to the $\mathrm{P}-\mathrm{O}$ vibration (noted $\mathrm{A}$ ), typical of $\mathrm{PW}_{12}$, and at $1180 \mathrm{~cm}^{-1}$ attributed to the $v_{\mathrm{C}-\mathrm{C}}+v_{\mathrm{C}-\mathrm{N}}$ vibrations (noted B), typical of the studied MOFs. The evolution of this ratio reveals that a maximum amount of $\mathrm{POM}$ immobilized into the MOF is reached for ca. $30 \mathrm{mg}$ of $(\mathrm{TBA})_{3} \mathrm{PW}_{12} \mathrm{O}_{40}$ in the reaction medium, corresponding to a $\mathrm{PW}_{12} / \mathrm{Zr}$ molar ratio of 0.05 (Fig. 3c). PW $_{12} @ Z_{6}$-TCPP-120 was also synthesized for further characterization purposes (see experimental section). The POM loading was evaluated by EDS (Table S1, ESI + ), suggesting an average of 1 POM every $2 Z_{r_{6}}$ units in the free-base $\mathbf{P W}_{\mathbf{1 2}} @ \mathbf{Z r}_{\mathbf{6}}$ TCPP-120 and 1 POM every $4 Z_{6} r_{6}$ units in the Fe-metallated PW $12 @ Z_{6}$-TCPP-Fe-120. C, H, N analyses indicate missing linkers in both cases, a consequence of the immobilization of the negatively charged POMs as well as of the presence of the PCN-224 phase.

In addition, $\mathrm{N}_{2}$ sorption isotherms (Fig. $\mathrm{S} 3, \mathrm{ESI}+$ ) evidence a decrease of the surface area of the POM@MOFs compounds when compared to that of the POM-free $\mathrm{Zr}_{6}$-TCPP-Fe-25 material, as expected from the incorporation of $\mathrm{PW}_{12}$ into the MOF's pores. More importantly, solid-state NMR ${ }^{31} \mathrm{P}\left\{{ }^{1} \mathrm{H}\right\}$ c)

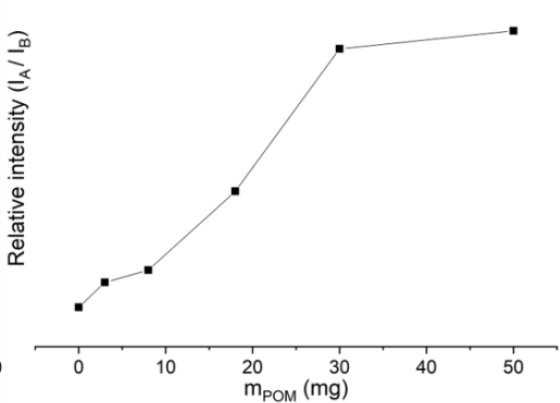

Fig. 3 Impact of a stepwise increase of the amount of PW 12 in the synthesis medium of PW $\mathbf{1 2}_{12} @ \mathbf{Z r}_{6}-\mathrm{TCPP}_{\mathbf{F}} \mathbf{F e}-\mathbf{1 2 0}$ on its (a) PXRD and (b) IR spectra where black dashed lines indicate bands characteristic of PW 12 while the red dashed line indicates a band of the MOF host. (c) Evolution of the ratio between the intensities of $A$ and $B$ bands (see text). 


\section{COMMUNICATION}

a)

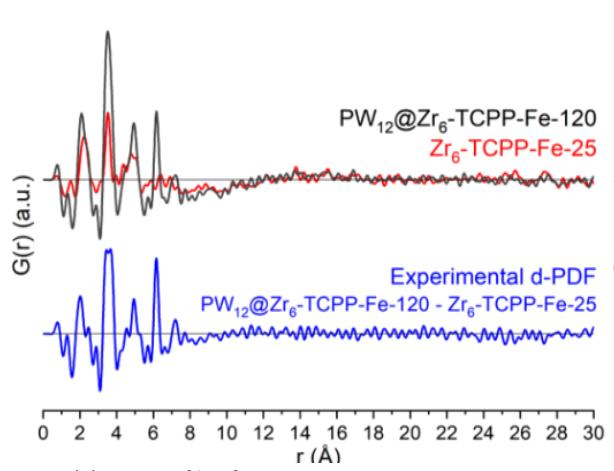

b)

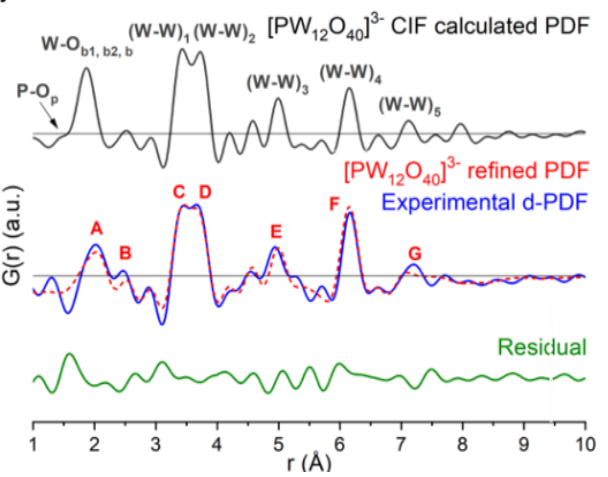

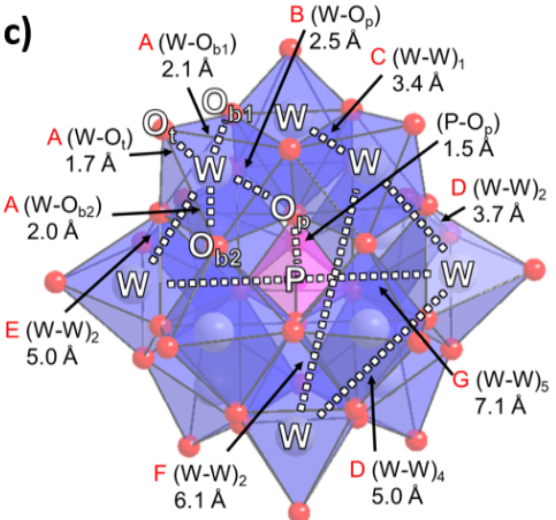

Fig. 4 (a) PDF profiles for PW $\mathrm{PW}_{12}$ from reported crystallographic data ${ }^{23}$ and the experimental d-PDF associated to PW $_{12}$ in PW $_{12} @ Z_{6}-\mathrm{TCPP}_{\mathbf{6}}-\mathrm{Fe}_{-120}$, superimposed with the refined d-PDF and residual profile. A$\mathrm{H}$ labels of peaks correspond to the indicated refined distances in the POM structure as illustrated in (c). $\mathrm{WO}_{6}$, blue octahedra; $\mathrm{PO}_{4}$, pink tetrahedron; $\mathrm{O}$, red sphere; $\mathrm{W}$, grey spheres; $P$, pink sphere.

CPMAS spectra of PW $12 @ Z r_{6}-$ TCPP-120 and PW $_{12} @ Z_{6}-r_{6}$ TCPPFe-120 (Fig. S4, ESI+) clearly show a characteristic single peak at $-15.9 \mathrm{ppm}$ and $-15.5 \mathrm{ppm}$, respectively, in the expected chemical shifts range for $\mathrm{PW}_{12}, 22$ thus suggesting the integrity of the POM. Moreover, the line broadening and appearance of spinning sideband in the spectrum of $\mathbf{P W}_{\mathbf{1 2}} @ \mathbf{Z r}_{\mathbf{6}}-\mathrm{TCPP}-\mathrm{Fe}-\mathbf{1 2 0}$ are due to chemical shift anisotropy and anisotropic bulk magnetic susceptibility broadening of ${ }^{31} \mathrm{P}$ nuclei in interaction with the Fe"l' paramagnetic centre.

In addition, PW $\mathbf{1 2} @ Z_{\mathbf{6}_{\mathbf{6}}}$-TCPP-Fe-120 and $\mathbf{P W}_{\mathbf{1 2}} @ \mathrm{Zr}_{\mathbf{6}}$-TCPP120 were studied using cyclic voltammetry (CV) by deposition on a glassy carbon electrode of a thin film of the composites (Fig. S5, ESI + ). The electrochemical signatures of $\mathrm{PW}_{12}{ }^{24}$ and FeTCPP ${ }^{12}$ in solution have been compared to those of the POM@MOF composites showing that not only they were in full agreement with those reported in the literature but also allowed to unambiguously attribute the characteristic redox peaks of these two components on the composite materials. To further investigate the structural features of these porphyrinbased MOFs, a Pair Distribution Function (PDF) analysis was undertaken. Reflecting the probability of finding two atoms separated by a distance $r$, PDF data provide quantitative information about the local atomic structure, while giving a global overview of the sample. They have proved to be particularly powerful in the field of MOFs recently. ${ }^{25}$ In a first step, considering we may have a mixture of structurally related MOFs, MOF-525 and/or PCN-224, difficult to distinguish using PXRD only, we investigated the use of PDF intending at identifying distinctive features related to each phase. This was undertaken using $\mathrm{Zr}_{6}$-TCPP-Fe-25 as a case study. As references, the PDF profiles of MOF-525 and PCN-224 were simulated using their DFT-optimized crystal structures. They exhibit common features due to their common $\mathrm{Zr}$-oxocluster but also distinctive particularities (see ESIt for details, Figs. S5-S8). The PDF profile of $\mathrm{Zr}_{6}$-TCPP-Fe-25 exhibits specific features from both MOFs (Fig. S9). On the one hand, it has strong similarities with that of
MOF-525, with a distinct peak at $3.1 \AA$ and a relatively intense peak at $5.1 \AA$, which are both a signature of $\mathrm{Zr}$-connected TCCP linkers. On the other hand, secondary features suggest the possible presence of $\mathbf{P C N}-\mathbf{2 2 4}$ in $\mathbf{Z r}_{\mathbf{6}}$-TCPP-Fe-25, such as a rather intense peak at $4.0 \AA$ (associated to $-\mathrm{OH}$ and $\mathrm{H}_{2} \mathrm{O}$ molecules in place of missing linkers) and a similar PDF profile to that of PCN-224 in the [5.5-7.5 $\AA$ ] radial distance range. Overall, this analysis indicates that $\mathbf{Z r}_{6}-\mathrm{TCPP}-\mathrm{Fe}-\mathbf{2 5}$ is unambiguously a mixture of MOF-525 and PCN-224.

In a second step, PDF data were further exploited to investigate the structural integrity of the immobilized POM within $\mathbf{P W}_{\mathbf{1 2}} @ \mathbf{Z r}_{\mathbf{6}}$ TCPP-Fe-120. Using the differential PDF method (d-PDF ${ }^{26}$ recently applied to characterize a POM@UiO-67 composite, ${ }^{10}$ the d-PDF of the immobilized $P_{12}$ was obtained by subtracting directly the PDF of $\mathbf{Z r}_{\mathbf{6}}-\mathbf{T C P P}-\mathbf{F e}-\mathbf{2 5}$ from that of $\mathbf{P W}_{\mathbf{1 2}} @ \mathbf{Z r}_{6}$ TCPP-Fe-120 (Fig. 4a and S9, see ESIt for details). The POM's dPDF profile is compared in the [1-10 $\AA]$ range to that of an isolated $\mathrm{PW}_{12} \mathrm{O}_{40}{ }^{3-}$ calculated from reported crystallographic data $^{23}$ (Fig. 4b). The two PDFs are indeed very similar allowing a precise assignment of all PDF peaks to characteristic W-O and $\mathrm{W}-\mathrm{W}$ distances and a refinement of the $\mathrm{PW}_{12}$ structure as illustrated in Fig. 4c (see ESIt for details, Fig S10, Table S2). The relatively good quality of the refinement $\left(R_{w}=29.3 \%\right)$ and the refined structure of $P W_{12}$ indicate that the $P O M$ is indeed fully preserved upon its immobilization in $\mathbf{Z r}_{6}$-TCPP-Fe-120.

To get an in-depth insight into the host-guest interactions at play between the $\mathrm{PW}_{12}$ and the MOF, Density Functional Theory (DFT) calculations were performed using MOF-525(Fe) phase as the main host. In a first step, simulated annealing (SA) calculations followed by dispersion-corrected DFT-D3 geometry optimizations were done to identify the most likely position of the POM in MOF-525(Fe) and qualify its host-guest interactions within the MOF. They reveal a non-centered positioning of the POM within the MOF's cubic cage showing $\mathrm{PW}_{12}$ cornered close to a $\mathrm{Zr}$-oxocluster inorganic node (Fig. 5). The interaction energy between the $\mathrm{PW}_{12}$ and MOF-525(Fe) was estimated $-422 \mathrm{~kJ} \mathrm{~mol}^{-}$ 
1 which corresponds to coordination-like interactions between the $\mathrm{O}_{\text {POM }}$ atoms and Fe ions of the TCCP linkers and dispersive interactions with $\mathrm{Cl}$ atoms, in addition to POM-TCCP hydrogen bonds (Fig. S12a). These results correlate well with 2D ${ }^{31} \mathrm{P}_{-}{ }^{1} \mathrm{H}$ correlation MAS NMR experiment (Fig. S12b) demonstrating the spatial proximity of the POMs to the inorganic nodes. Overall, DFT calculations coupled with solid-state NMR added remarkable insights on the POM-MOF interactions to PDF analysis.

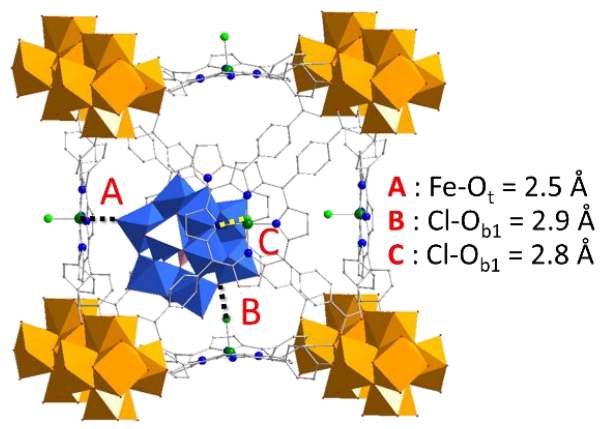

Fig. 5 Most likely positioning of the POM inside the hybrid framework obtained by DFT calculations.

In conclusion, targeting porphyrinic POM@MOF systems, the addition of the $\mathrm{PW}_{12}$ polyoxometalate in the synthetic medium of porphyrin MOFs was explored using a high temperature one-pot synthetic protocol. In the absence of POM, a mixture of the kinetic MOF-525/PCN-224 and thermodynamic MOF-545 topologies are obtained. Remarkably, the addition of $\mathrm{PW}_{12}$ in the synthetic medium prevents the formation of the expected thermodynamic MOF$\mathbf{5 4 5}$ phase while favoring the formation of the kinetic ones even at high temperature, pointing towards the structure-directing role of the POM. Simultaneously we developed in-depth characterization methods of $\mathbf{P W}_{\mathbf{1 2}} @$ MOF by utilizing differential Pair Distribution Function as a direct structural signature of the POM, solid-state NMR and DFT calculations. They showed unequivocally the structural integrity of the POM inside the porphyrinic MOF along with an insight on the hostguest interactions between the different components, circumventing the difficulties inherent to the Rietveld refinement of powder X-ray diffraction data of such long-range disordered materials. These results open the way for more complete research on the templating effect of POMs on the topology of porphyrinic MOFs and their effect on their catalytic properties.

This work was supported by CNRS, UVSQ, the Ministère de l'Enseignement supérieur, de la Recherche et de I'Innovation, the French National Research Agency (ANR) as part of the 'Investissements d'Avenir' program n ${ }^{\circ}$ ANR-11-IDEX-0003-02 and CHARMMMAT ANR-11-LABX-0039. We also acknowledge financial support from the Paris lle-de-France Region DIM 'Respore'. The IMAP laboratory is gratefully acknowledged for $\mathrm{N}_{2}$ porosimetry measurements. The calculations have been performed using the HPC re-sources from GENCI (CINES) through Grant 2016-097343.

\section{Conflicts of interest}

There are no conflicts to declare.

\section{Notes and references}

1 A. Bavykina, N. Kolobov, I. S. Khan, J. A. Bau, A. Ramirez and J. Gascon, Chem. Rev.

2 P. Horcajada, R. Gref, T. Baati, P. K. Allan, G. Maurin, P. Couvreur, G. Férey, R. E. Morris and C. Serre, Chem. Rev., 2012, $112,1232-1268$.

3 A. Kirchon, L. Feng, H. F. Drake, E. A. Joseph and H.-C. Zhou, Chem. Soc. Rev., 2018, 47, 8611-8638.

4 Z.-Y. Gu, J. Park, A. Raiff, Z. Wei and H.-C. Zhou, ChemCatChem, 2014, 6, 67-75.

5 W.-Y. Gao, M. Chrzanowski and S. Ma, Chem. Soc. Rev., 2014, 43, 5841-5866.

6 W. Morris, B. Volosskiy, S. Demir, F. Gándara, P. L. McGrier, H. Furukawa, D. Cascio, J. F. Stoddart and O. M. Yaghi, Inorg. Chem., 2012, 51, 6443-6445.

7 D. Feng, W.-C. Chung, Z. Wei, Z.-Y. Gu, H.-L. Jiang, Y.-P. Chen, D. J. Darensbourg and H.-C. Zhou, J. Am. Chem. Soc., 2013, 135, 17105-17110.

8 J.-S. Qin, S. Yuan, C. Lollar, J. Pang, A. Alsalme and H.-C. Zhou, Chem. Commun., 2018, 54, 4231-4249.

9 G. Paille, M. Gomez-Mingot, C. Roch-Marchal, B. Lassalle-Kaiser, P. Mialane, M. Fontecave, C. Mellot-Draznieks and A. Dolbecq, J. Am. Chem. Soc., 2018, 140, 3613-3618.

10 Y. Benseghir, A. Lemarchand, M. Duguet, P. Mialane, M. GomezMingot, C. Roch-Marchal, T. Pino, M.-H. Ha-Thi, M. Haouas, M. Fontecave, A. Dolbecq, C. Sassoye and C. Mellot-Draznieks, J. Am. Chem. Soc., 2020, 142, 9428-9438.

11 D. Li, M. Kassymova, X. Cai, S.-Q. Zang and H.-L. Jiang, Coord. Chem. Rev., 2020, 412, 213262.

12 I. Hod, M. D. Sampson, P. Deria, C. P. Kubiak, O. K. Farha and J. T. Hupp, ACS Catal., 2015, 5, 6302-6309.

13 H. Zhang, J. Wei, J. Dong, G. Liu, L. Shi, P. An, G. Zhao, J. Kong, X. Wang, X. Meng, J. Zhang and J. Ye, Angew. Chem. Int. Ed., 2016, 55, 14310-14314.

14 X. Gong, H. Noh, N. C. Gianneschi and O. K. Farha, J. Am. Chem. Soc., 2019, 141, 6146-6151.

15 M. L. Kelty, W. Morris, A. T. Gallagher, J. S. Anderson, K. A. Brown, C. A. Mirkin and T. D. Harris, Chem. Commun., 2016, 52, 7854-7857.

16 S. M. Shaikh, P. M. Usov, J. Zhu, M. Cai, J. Alatis and A. J. Morris, Inorg. Chem., 2019, 58, 5145-5153.

17 P. Deria, J. Yu, R. P. Balaraman, J. Mashni and S. N. White, Chem. Commun., 2016, 52, 13031-13034.

18 P. Deria, D. A. Gómez-Gualdrón, I. Hod, R. Q. Snurr, J. T. Hupp and O. K. Farha, J. Am. Chem. Soc., 2016, 138, 14449-14457.

19 M. R. DeStefano, T. Islamoglu, S. J. Garibay, J. T. Hupp and O. K. Farha, Chem. Mater., 7.

20 H. Noh, C.-W. Kung, T. Islamoglu, A. W. Peters, Y. Liao, P. Li, S. J. Garibay, X. Zhang, M. R. DeStefano, J. T. Hupp and O. K. Farha, Chem. Mater., 2018, 30, 2193-2197.

21 G. Kickelbick, P. Wiede and U. Schubert, Inorganica Chim. Acta, 1999, 7.

22 W. Salomon, C. Roch-Marchal, P. Mialane, P. Rouschmeyer, C. Serre, M. Haouas, F. Taulelle, S. Yang, L. Ruhlmann and A. Dolbecq, Chem. Commun., 2015, 51, 2972-2975.

23 A. Kremenović, A. Spasojević-de Biré, R. Dimitrijević, P. Sciau, U. B. Mioč and P. Colomban, Solid State Ion., 2000, 132, 39-53. 
Journal Name

24 K. Eda and T. Osakai, Inorg. Chem., 2015, 54, 2793-2801.

25 C. Castillo-Blas, J. M. Moreno, I. Romero-Muñiz and A. E.

Platero-Prats, Nanoscale, DOI:10.1039/DONR01673J.

26 K. W. Chapman, P. J. Chupas and C. J. Kepert, J. Am. Chem. Soc., 2005, 127, 11232-11233. 